\title{
Aspirin for primary prevention of cardiovascular disease: Advice for a decisional strategy based on risk stratification
}

\author{
(1D) Alberto Aimo, (1) Raffaele De Caterina
}

Department of Cardiology, University Hospital of Pisa; Pisa-Italy

\section{ABSTRACT}

The need for aspirin therapy as part of primary prevention of cardiovascular (CV) disease is currently being highly debated, especially after 3 studies in different settings reported that a reduction in ischemic events is largely counterbalanced by an increase in bleeding events. One possible explanation for these results is the progressive reduction in the risk of major adverse cardiovascular events (MACE) as a result of primary prevention, which has accompanied global education programs that have led to patients smoking less, exercising more, and increasingly undertaking lipid-lowering therapies. Based on a meta-regression of the benefits and harmful effects of aspirin therapy in primary prevention as a function of the 10-year risk of MACE, we favor a differentiated and personalized approach that acknowledged differences between patients and emphasized an individualized assessment of benefits and risks. Following general preventive measures (physical exercise, cessation of smoking, treatment of hypertension and hypercholesterolemia, etc.), an individualized approach to prescribing aspirin is still warranted. When patients are less than 70 years of age, clinicians should assess the 10-year CV risk. Aspirin treatment should be considered only when the CV risk is very high and the bleeding risk is low, after taking into account the patient's preferences. (Anatol $J$ Cardiol 2020; 23: 70-8)

Keywords: aspirin, primary cardiovascular prevention, bleeding, major acute cardiovascular events, MACE, benefit-risk balance

\section{Introduction}

Cardiovascular (CV) disease prevention has been defined as "a coordinated set of actions, at the population level or targeted at an individual, that are aimed at eliminating or minimizing the impact of CV diseases and their related disabilities" (1). Primary CV prevention consists of the prevention of symptom development (chronic coronary syndrome) or acute coronary syndrome events in asymptomatic individuals. The need for aspirin therapy as part of primary prevention of CV disease is currently being highly debated, especially after 3 studies in different settings reported that a reduction in ischemic events is largely counterbalanced by an increase in bleeding events (2-6). These results have been pooled together with those from previous trials (7-9) and have prompted an intense debate among leading experts in the field (10-14), which have culminated in changes being made to the European and American CV recommendations $(15,16)$. Although the role of aspirin for primary prevention has been proposed to be residual in an era marked by dwindling CV risk (at least in Europe and the US) (14), and the idea that aspirin should never be prescribed for primary prevention (given the absence of a demonstrated net clinical benefit) has been hastily put forward (17), the prevailing notion is that aspirin therapy should be reserved to the still-existing subgroup of individuals undergoing primary prevention in whom the ischemic risk outweighs the bleeding risk $(11,12,18)$. The same concept emerges from the most recent guideline recommendations $(15,16)$ and from some algorithms that aim to support clinical decisions $(12,18)$. Clinical cardiologists are now faced with an evolving landscape of recommendations and expert opinions that broadly advocate a tailored approach to aspirin prescription, based on an individualized assessment of the ischemic and bleeding risk. However, these varied opinions may often generate uncertainties regarding the need for aspirin therapy in individual cases.

In the present review, we reappraise the recent advances on aspirin for primary prevention, discuss a recently proposed decisional strategy (18), and try to envisage the research-related developments of in this field.

Address for correspondence: Raffaele De Caterina, MD, Department of Cardiology,

University Hospital of Pisa; Pisa-Italy

E-mail: raffaele.decaterina@unipi.it

Accepted Date: 01.11.2019 Available Online Date: 18.12.2019

(C) Copyright 2020 by Turkish Society of Cardiology - Available online at www.anatoljcardiol.com DOl:10.14744/AnatolJCardiol.2019.89916 


\section{The latest trials}

\section{Aspirin to Reduce Risk of Initial Vascular Events (ARRIVE) trial}

The ARRIVE trial was a randomized, double-blind, placebocontrolled, multi-center study that enrolled men aged $\geq 55$ years and women aged $\geq 60$ years with a 10 -year risk of major adverse cardiovascular events (MACE), ranging from $10 \%$ to $20 \%$, i.e., the expected moderate CV risk (2). Patients at high risk of bleeding and those with diabetes were excluded. The patients $(n=12,546)$ were randomized on the basis of a 1:1 ratio of $100 \mathrm{mg}$ of daily aspirin to a placebo. Over a median 60-month follow-up, there was no significant difference in the primary endpoint [a composite of time to first myocardial infarction (MI), stroke, CV death, unstable angina, or transient ischemic attack], which recorded a hazard ratio (HR) of 0.96 and a $95 \%$ confidence interval (Cl) of 0.81-1.13 $(p=0.604)$. Similarly, the incidence rates of both fatal and nonfatal myocardial MI were not significantly different. With respect to safety, gastrointestinal (GI) bleeding events were more frequent in the aspirin groups than in the placebo groups ( $H R=2.11,95 \%$ $\mathrm{Cl}=1.36-3.28 ; \mathrm{p}<0.001$ ), although these events were predominantly mild. The incidence of serious adverse events was similar in both treatment groups $(20.19 \%$ vs. $20.89 \%$ in the aspirin and placebo groups, respectively) (2).

Although designed as a study exploring an "intermediate risk" population, the observed incidence of MACE in ARRIVE was significantly lower (4\%) than predicted. As such, we believe that the negative benefit-to-risk ratio observed in ARRIVE is informative for contemporary "low-risk" patients, but should not be generalized for those at substantially higher risk.

\section{A Study of Cardiovascular Events in Diabetes (ASCEND) trial}

Patients with diabetes mellitus have a substantially higher risk of experiencing an atherothrombotic event first, as compared to non-diabetic subjects, but clinical trials with aspirin in this setting have proven to be inconclusive (19).

The ASCEND trial enrolled 15,480 patients aged $\geq 40$ years, with any form of diabetes but no symptomatic CV disease at the baseline. These patients were randomized to be administered with either $100 \mathrm{mg}$ of aspirin daily or a placebo, and also omega-3 fatty acids vs. placebo. The mean patient age was 63 years and the gender distribution included $63 \%$ males. About $94 \%$ patients had type 2 diabetes, with median disease duration of 7 years. Other CV risk factors often coexisted with diabetes, with $62 \%$ of patients affected by hypertension and $75 \%$ being on statins, with a mean body mass index in the obese range. The primary efficacy outcome was a composite of nonfatal MI, non-hemorrhagic stroke, transient ischemic attack, or CV death. The primary safety outcome was major bleeding. Over a mean follow-up duration of 7.4 years, the primary efficacy endpoint occurred in a lesser percentage of participants in the aspirin group than in the placebo group [rate ratio (RR): $0.88,95 \% \mathrm{Cl}: 0.79-0.97 ; p=0.01]$. Major bleeding events occurred more frequently in the aspirin group (RR: $1.29,95 \% \mathrm{Cl}$ :
$1.09-1.52 ; p=0.003)$, with most of the excesses represented by GI bleeding and other extracranial bleeding (3).

In no patient subgroups did the benefits clearly outweigh the risks, including in the highest-risk subgroups, however, as in ARRIVE, the overall risk was low-to-moderate, with only $17.2 \%$ of patients having a 5 -year risk exceeding $10 \%$ (3). Interestingly, although around half of the excesses of bleeding events occurred in the GI tract, "even near the end of the trial in 2016, only approximately one quarter of participants were receiving protonpump inhibitors (PPIs)" (3). Therefore, it was concluded that the systematic prescription of a PPI could increase the net clinical benefit of aspirin treatment.

\section{Aspirin in Reducing Events in the Elderly (ASPREE) trial}

The ASPREE trial was a randomized, placebo-controlled trial that involved Australian and American centers and investigated whether aspirin therapy would prolong the lives of healthy community-dwelling older adults and keep them free from dementia and physical disability. The trial included subjects aged $\geq 70$ years (or $\geq 65$ years among blacks and Hispanics from the US) who were free from life-limiting illness and had no documented vascular disease. Having a high bleeding risk was the exclusion criterion. Following a run-in phase to exclude patients with unsatisfactory compliance to treatment, 19,114 subjects were enrolled and randomized in a 1:1 ratio of aspirin to placebo. The median patient age was 74 years, $56 \%$ of patients were women, $11 \%$ had diabetes, and another $11 \%$ had undergone aspirin therapy previously. The trial was terminated after a median 4.7-year follow-up, and provided no evidence of benefit from aspirin with regard to the primary endpoint, which was a composite of death, onset of dementia, and persistent physical disability ( $\mathrm{HR}=1.01,95 \% \mathrm{Cl}=0.92-1.11 ; \mathrm{p}=0.79$ ). There was a trend toward increased mortality among patients on aspirin (HR=1.14, 95\% $\mathrm{Cl}=1.01-1.29)$, largely because of a higher incidence of cancer-related death and not achieving statistical significance when accounting for the multiplicity of secondary endpoints. This finding for cancer is inconsistent with prior reports. With regard to CV disease, findings from ASPREE were neutral for incident events $(\mathrm{HR}=0.95,95 \% \mathrm{Cl}=0.83-1.08)$ with a consistent increase in major hemorrhage $(\mathrm{HR}=1.38,95 \% \mathrm{Cl}=1.18-1.62$; $p<0.001)$. Healthy elderly patients thus do not seem to benefit from aspirin in primary prevention (4-6).

\section{Meta-analyzes on aspirin for primary prevention}

The results from the three latest trials, summarized in Table 1 , have been pooled together with previous studies. First, Dr. Ridker (14) observed that no trial has ever shown a benefit of reduced mortality from aspirin and that a net benefit on the hard clinical endpoint is unlikely to emerge in an era when the approach to primary CV prevention is increasingly aggressive. A meta-analysis evaluated 11 trials with 157,248 individuals and excluded studies enrolling subjects with evidence of subclinical vascular disease, such as a reduced ankle-brachial index (ABI) (7). At a mean follow-up of 6.6 years, aspirin was not associated 


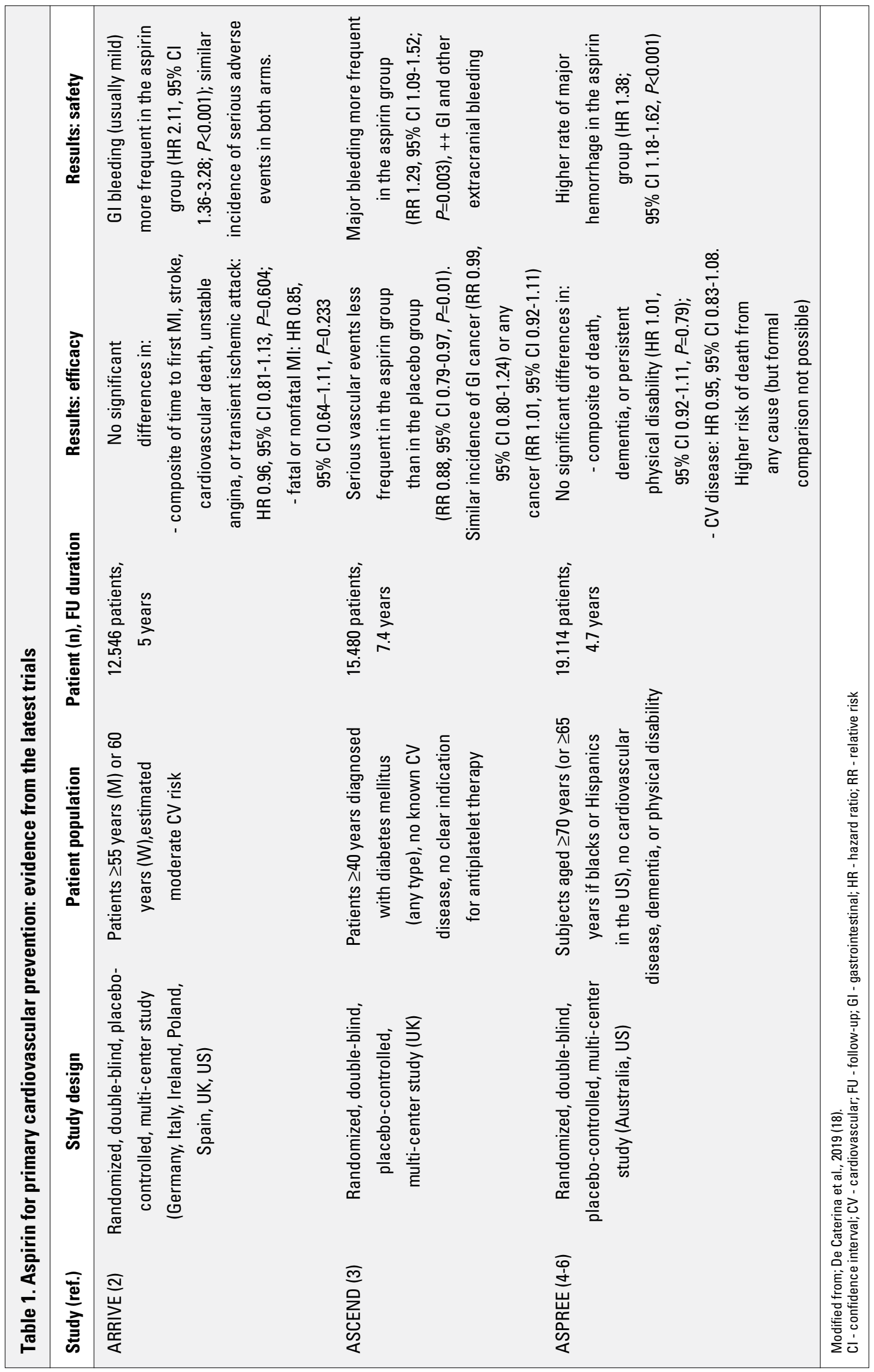


with a lower incidence of all-cause mortality (RR: $0.98,95 \% \mathrm{Cl}$ : 0.93-1.02; $p=0.30$ ), but was associated with a higher incidence of major bleeding (RR: $1.47,95 \% \mathrm{Cl}: 1.31-1.65 ; \mathrm{p}<0.001$ ) and intracranial hemorrhage (RR: $1.33,95 \%$ Cl: $1.13-1.58 ; p=0.001$ ). A similar effect on all-cause mortality and major bleeding was demonstrated in diabetic patients and those deemed to have a high $\mathrm{CV}$ risk (as those with a 10 -year risk of $>7.5 \%$ were considered). Aspirin was associated with a lower incidence of myocardial infarction (RR: $0.82,95 \% \mathrm{Cl}: 0.71-0.94 ; \mathrm{p}=0.006$ ), although this effect did not emerge from the more recent trials (7).

Another meta-analysis used a broader definition of primary prevention ("participants without known preexisting CV disease") (8), thus including trials with asymptomatic atherosclerotic disease, such as the Prevention of Progression of Arterial Disease and Diabetes (POPADAD) (20) and Aspirin for Asymptomatic Atherosclerosis (AAA) trials (21). A total of 13 trials randomizing 164,225 participants with a median baseline risk of the primary CV outcome was $9.2 \%$ (range: $2.6-15.9 \%$ ). For 256 subjects needed to be harmed, aspirin use was associated with significant reductions in the composite CV outcome (a composite of CV mortality, nonfatal myocardial infarction, and nonfatal stroke) as compared to no aspirin administration (HR: $0.89,95 \% \mathrm{Cl}: 0.84-0.95)$. The use of aspirin was not associated with lower all-cause mortality (HR: 0.94, 95\% Cl: 0.88-1.01) or CV mortality (HR: $0.94,95 \%$ Cl: $0.83-1.05$ ) as compared to no aspirin administration. Furthermore, the use of aspirin was associated with a reduction in myocardial infarction (HR: $0.85,95 \%$ Cl: $0.73-0.99$ ) and ischemic stroke (HR: $0.81,95 \% \mathrm{Cl}$ : $0.76-0.87)$, and conversely, it was associated with an increased risk of major bleeding events (HR: $1.43,95 \% \mathrm{Cl} 1.30-1.56$ ), when the number needed to be harmed was 210 (8).

The efficacy and safety of aspirin in patients with diabetes were evaluated in a dedicated meta-analysis that considered data from 10 trials and 33,679 patients. Aspirin did not significantly reduce the risk of MACE ( $\mathrm{RR}=0.93,95 \% \mathrm{Cl}=0.87-1.00, \mathrm{p}=0.06)$, CV mortality ( $\mathrm{RR}=0.95,95 \% \mathrm{Cl}=0.83-1.09, \mathrm{p}=0.49$ ), myocardial infarction ( $\mathrm{RR}=0.91,95 \% \mathrm{Cl}=0.75-1.11, \mathrm{p}=0.36)$, or stroke ( $\mathrm{RR}=0.91$, $95 \% \mathrm{Cl}=0.76-1.10, \mathrm{p}=0.33)$, however, there was a small but significant increase in the risk of all bleeding events ( $R R=1.29,95 \%$ $\mathrm{Cl}=1.07-1.55, \mathrm{p}=0.01$ ) (9). Given the $\mathrm{p}=0.06$ value for MACE prevention by aspirin, this meta-analysis confirms the equivalence between the prevention of ischemic events and an increase in bleeding events proposed by the ASCEND trial (3).

\section{Subclinical atherosclerosis and diabetes as risk modifiers}

As noted above, the 3 meta-analyzes also tried to examine whether the safety and efficacy of aspirin were influenced by either subclinical atherosclerotic disease or diabetes. Indeed, the increased coronary artery calcium score, atherosclerotic plaques determined by carotid artery scanning, and reduced $A B I$ are cited in ESC guidelines as risk modifiers (1), and patients with diabetes and no established CV disease are deemed to have a moderate or high CV risk (15). Based on these premises, one would expect a more favorable risk-to-benefit ratio of aspirin therapy in these settings. Nonetheless, no evidence from randomized controlled trials is currently available on patients with asymptomatic carotid disease or coronary artery calcifications. The POPADAD and AAA trials did not find a benefit from aspirin therapy in patients with reduced $A B I(20,21)$ and no net benefit from aspirin emerged in the case of diabetic patients. Consequently, current guidelines do not recommend aspirin therapy in asymptomatic patients with evidence of atherosclerotic disease or diabetes, with the exception of carotid artery stenosis $>50 \%$ [class Ila, level of evidence (LOE): C] (22), due to its established prognostic value for ischemic stroke and myocardial infarction (23).

In our opinion, it would be more reasonable to perform a global assessment of CV risk and to decide the need for aspirin therapy on that basis, instead of focusing on single variables and cutoffs (such as the presence of a $>50 \%$ carotid stenosis) that will always inevitably remain arbitrary.

\section{Therapeutic algorithms based on risk stratification}

In 2014, an international panel of experts published a consensus document advocating a tailored strategy for aspirin prescription with regards to primary CV prevention (24). From all the trials available at that time, the authors extrapolated the relative benefit or harm from aspirin versus placebo as a function of the 10-year risk of MACE in the control group. This analysis suggested that the relative benefit from aspirin (in terms of reduced ischemic events) increases progressively and as a parallel with the 10-year risk of MACE, whereas the relative risk of major bleeding remains unchanged. A substantial proportion of major bleedings had a GI origin, which is worth noting, as the risk of $\mathrm{GI}$ bleeding can be substantially reduced by therapy with PPIs. The authors concluded that the reduction in thromboembolic events was balanced by an increased risk of bleeding in patients who were at low risk of MACE, while patients with a higher risk seemed to derive a net benefit from aspirin. As cutoff points useful for clinical decisions, the $10 \%$ and $20 \%$ values of the 10-year risk of MACE were selected. Therefore, aspirin for primary prevention was not to be prescribed when the estimated 10 -year risk of MACE was $<10 \%$, but might be considered when the risk was $10-20 \%$, and was advised when the 10 -year risk was $>20 \% 24$. Further elements to be considered were the patient's bleeding risk and family history of GI cancer, particularly colorectal cancer, since epidemiologic evidence indicates that regular and long-term use of aspirin is associated with a lower incidence of colorectal cancer (25).

We have updated the same analysis following the publication of the latest trials on aspirin for primary prevention (Fig. 1) $(2-6,18)$. The correlation between the 10-year risk of MACE and the relative reduction in ischemic events is weaker than in the 2014 diagram ( $R$-values $=0.691$ vs. 0.729 , $p$-values $<0.001$ vs. 0.006 , respectively). Furthermore, the slope of the line depicting the relative reduction in ischemic events appears driven by the Early Treatment Diabetic Retinopathy Study (ETDRS) (26), an old trial that included patients either in primary or secondary prevention, 


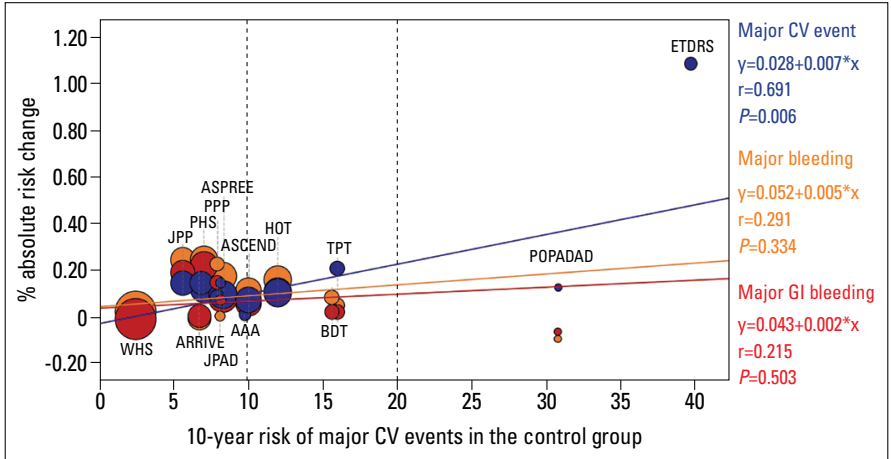

Figure 1. Relationships between the magnitude of antithrombotic benefit, bleeding risk, and cardiovascular risk in clinical trials of aspirin for primary prevention. This univariate linear regression reports the effect of aspirin as a function of the baseline cardiovascular (CV) risk. The regression lines correspond to major CV events (vascular death, nonfatal myocardial infarction, and nonfatal stroke, excluding transient ischemic attacks or need for revascularization), major bleeding, and major gastrointestinal (GI) bleeding. The independent variable is the risk of major CV events per 10 patient-years in the control group of each trial. On the $y$-axis, the percentage absolute risk change is provided; for major CV events, this is calculated as risk/follow-up years in the control group minus the risk/years in the aspirin group, while for major bleeding and major GI bleeding it is calculated as risk/ follow-up years in the aspirin group minus the risk/years in the control group. Study weight is proportional to patient number. Each study is represented by three circles (one for each endpoint), each of whose size is proportional to patient number. Only one and two circles are reported for the Early Treatment Diabetic Retinopathy Study (ETDRS) (26) and the US Physicians Health Study (PHS) (37), respectively, since the data reported in the studies do not allow a complete evaluation of the bleeding risk

AAA - Aspirin for Asymptomatic Atherosclerosis (21); ARRIVE - Aspirin to Reduce Risk of Initial Vascular Events (2); ASCEND - A Study of Cardiovascular Events in Diabetes (3); ASPREE - Aspirin in Reducing Events in the Elderly (4-6); BDT - British Doctors Trial (38); HOT - Hypertension Optimal Treatment (39); JPAD - Japanese Primary Prevention of Atherosclerosis With Aspirin for Diabetes (40); JPPP Japanese Primary Prevention Project (41); PHS - Physician Health Study (37); POPADAD - Prevention of Progression of Arterial Disease and Diabetes (20); PPP - Primary Prevention Project (42); TPT - Thrombosis Prevention Trial (43); WHS Women's Health Study (44)

Modified from De Caterina et al., 2019 (18)

and the statistical significance is lost when this trial is excluded $(\mathrm{p}=0.085)$. We may also note that only 2 trials stand above the $20 \%$ risk threshold and that the reduction in ischemic events appears much more prominent in the ETDRS (26) than in the POPADAD trial (20), even though no bleeding events were reported in the ETDRS study (26). Other possible limitations of this analysis are the high degree of heterogeneity of trials on aspirin for primary prevention, including the much more intensive control of CV risk factors in most recent trials and the assessment of MACE instead of harder endpoints, such as fatal atherosclerotic events, the risk of which is estimated in the ESC SCORE charts (see below).

Despite these cautionary steps, the diverging slopes of the lines representing the relative benefit and risk of aspirin therapy seem to corroborate the conclusions of the 2014 consensus doc- ument. A revised version of the decisional algorithm is reported in Figure 2. Patients under primary CV prevention should achieve optimal control of CV risk factors, primarily targeting the recommended low-density lipoprotein and blood pressure levels (1, 27,28 ). Afterwards, when the patients reach an age of $<70$ years and are free from physical disability or dementia, they should undergo a stratification of their CV risk, particularly when there is evidence of subclinical atherosclerotic disease or diabetes. When the 10-year risk of MACE is $>20 \%$, there is a rationale for starting therapy with aspirin, after discussing the risks and benefits with the patient and particularly if there are no conditions of

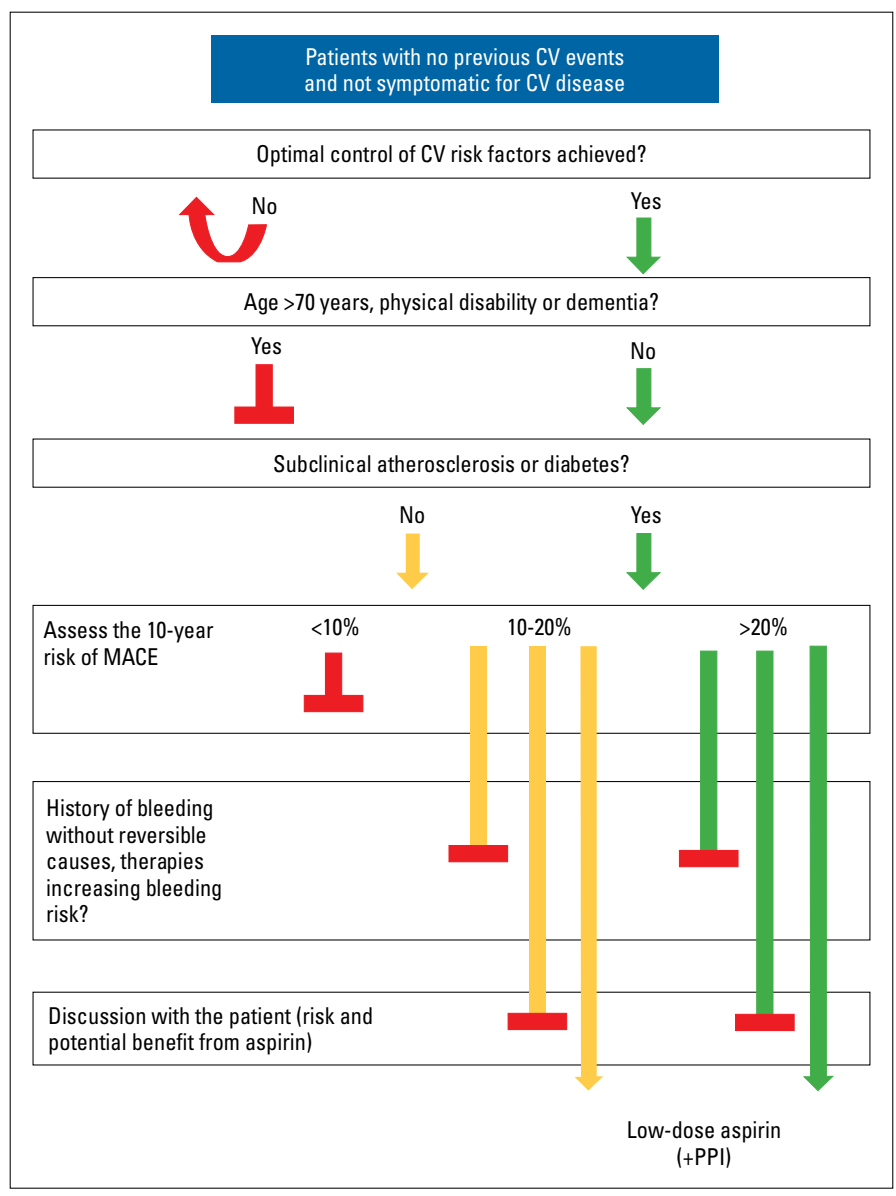

Figure 2. Proposed stepwise approach to prescribing aspirin for primary cardiovascular prevention

Patients undergoing primary cardiovascular (CV) prevention should achieve optimal control of CV risk factors. When patients are aged $<70$ years and are free from physical disability or dementia, they should undergo a CV risk stratification, especially when there is evidence of subclinical atherosclerosis or diabetes. When the 10 -year risk of major adverse cardiovascular events (MACE) is $>20 \%$, it is recommended to start therapy with aspirin after discussing the risks and benefits of aspirin intake with the patients, particularly if there are no conditions of increased bleeding risk. Aspirin should be prescribed along with a proton-pump inhibitor (PPI) to reduce the risk of gastrointestinal bleeding. The commencement of aspirin therapy should be carefully evaluated when the 10 -year risk is between $10 \%$ and $20 \%$, and aspirin should not be prescribed when the 10 -year risk is $<10 \%$ Modified from De Caterina et al., 2019 (18) 
increased bleeding risk. Aspirin should be most often prescribed along with a PPI to reduce the risk of GI bleeding. Aspirin therapy should be more carefully evaluated when the 10-year risk is 10 $20 \%$ and should not be prescribed when the 10 -year risk is $<10 \%$. Importantly, all patients must be thoroughly informed about the risks and potential benefits from this therapy.

A similar stepwise approach based on risk stratification has been recently proposed by Chiang et al. (12). The decision as to start, continue, or stop aspirin for primary prevention must follow a discussion with the patient in which the clinician is asked to first assess the patient's understanding and eagerness to engage in discussion regarding aspirin therapy (step 1), review potential benefits and harms from aspirin (step 2), ascertain the patient's preferences by asking questions that deal with the patient's familiarity on conditions prevented by aspirin and its adverse effects, and finally assess the patient's willingness to continue this medication long-term (step 3). When these steps are taken, aspirin should be started or continued when there is a combination of high CVD risk, a low bleeding risk, and the patient's preference to avoid CVD events. As general indications, the authors suggest that therapy be initiated when the 10-year risk is $>15 \%$ and that therapy be continued when the risk is $>10 \%$ and the patient is on aspirin for $>10$ years. Risk thresholds for treatment could be lower when the bleeding risk is low and when there is a strong patient preference for reducing the risk of CVD events. The relative benefit and risk of aspirin therapy should be carefully weighed in most elderly patients (e.g., patients aged $>75$ years). Finally, aspirin should not be prescribed or be discontinued when the 10 -year risk is $<5 \%$, there is a high risk of bleeding, or if the patient expresses a clear preference to avoid bleeding. The authors also recommend optimal control of $\mathrm{CV}$ risk factors as a necessary prerequisite for risk estimation and decision-making about aspirin therapy (12).

\section{Guideline recommendations}

The notion that decisions about aspirin therapy should be taken by an individualized assessment of ischemic and bleeding risks is corroborated by guidelines published in 2019. While the 2016 European Society of Cardiology (ESC) guidelines on CV disease prevention adopted the extreme position of advising against aspirin therapy in every patient ["Antiplatelet therapy is not recommended in individuals without CV disease due to the increased risk of major bleeding" (class III, LOE B) and "antiplatelet therapy (e.g., with aspirin) is not recommended for people with DM who do not have CVD" (class III, LOE A) (1)], the 2019 ESC guidelines on diabetes recommend that "In patients with DM at high/very high risk, aspirin $(75-100 \mathrm{mg} /$ day) may be considered in primary prevention in the absence of clear contraindications" (class IIb, LOE A), whereas "In patients with diabetes at moderate CV risk, aspirin for primary prevention is not recommended" (class III, LOE B). It is even specified that "When low-dose aspirin is used, PPIs should be considered to prevent GI bleeding" (class Ila, LOE A) (15). In the broader setting of primary prevention, the 2019 American College of Cardiology/ American Heart Association (ACC/AHA) guidelines recommend that "Low-dose aspirin (75-100 mg orally daily) [...] be considered for the primary prevention of ASCVD among select adults of 40 to 70 years of age who are at higher CV risk but not at increased bleeding risk" (class IIb, LOE A), whereas it should not be administered on a routine basis in individuals aged $>70$ years (class III, LOE B) and should be avoided in individuals of any age at increased risk of bleeding (class III, LOE C) (16).

\section{The challenge of risk prediction}

The necessary prerequisite for every approach based on risk stratification is the availability of measures that can accurately measure the patient's risk. This may be seen as the Achilles' heel of the proposed algorithms and guideline recommendations. Indeed, there is a plethora of risk scores to estimate the risk of CV events, but these scores are burdened by many limitations. For example, American- and European-based scores should be preferentially used in the respective geographical settings, but the ESC Systematic Coronary Risk Evaluation (SCORE) Risk Charts yield only the 10 -year risk of fatal atherosclerotic events, whereas the total CV event risk should be around 3 times higher for men, about 4 times higher for women, and lower in older persons, in whom a first event is more likely to be fatal (1). In contrast, the scores defined in a Framingham population evaluate both fatal and nonfatal events, but analyze highly heterogeneous endpoints [10-year risk of myocardial infarction or coronary death (29), coronary disease, cerebrovascular disease, peripheral vascular disease and heart failure (30), or atherosclerotic CV disease (31)]. Only this last endpoint, estimated by the Atherosclerotic CV Disease Risk Algorithm (31), can be assimilated to MACE. Moreover, all risk scores assess a very limited number of variables, some of them cannot be applied to patients with diabetes $(1,29)$, while others simply stratify patients according to the presence or absence of diabetes, despite the large variability in CV risk according to the type of diabetes, disease duration, organ damage, etc. (1). Additionally, while the $\mathrm{CV}$ risk decreases rapidly as a result of an increasingly intensive management of CV risk factors (32), Framingham-based scores date back to the '90s and 2000s. The striking discrepancy between expected and actual CV risks in the ARRIVE trial (where a combination of "various European and US risk calculators" was used) (2) lends some support to the hypothesis that risk scores, particularly the less recent American scores, tend to overestimate CV risk. Keeping a perspective of a decisional strategy that is based on accurate risk stratification, we can envisage a dedicated assessment of the prognostic performance of Framingham-based risk score and a perform a search for new risk scores that can include a greater number of risk factors and possibly use technology-based tools, such as artificial intelligence and deep learning (33-35).

Accurate estimation of the bleeding risk is equally important, but has received little attention so far. The main evidence de- 
rives from a prospective cohort study from a primary care setting in New Zealand (36). Here 385,191 persons aged 30-79 years to whom aspirin was prescribed for primary prevention were evaluated from 2007 to 2016. A prognostic model that included demographic characteristics (age, ethnicity, and socioeconomic deprivation), clinical measurements (systolic blood pressure and ratio of total-high-density lipoprotein cholesterol), family history of premature CVD, medical history (smoking, diabetes, bleeding, peptic ulcer disease, cancer, chronic liver disease, chronic pancreatitis, or alcohol-related conditions), and medication use (nonsteroidal anti-inflammatory agents, corticosteroids, and selective serotonin reuptake inhibitors) was developed, and showed good calibration for the prediction of major bleeding (36). The validation of this model in other settings may be worthwhile for further study.

\section{Introducing the notion of weighed net clinical benefit}

In the diagram reported in Figure 1, the relative decrease of MACE is compared to the relative increase in major bleeding for all values of the 10-year risk, following which an equipoise between MACE and major bleeding events is proposed. A more sophisticated evaluation of the net clinical benefit would attribute different weights to the single ischemic and bleeding endpoints according to their impact on morbidity and mortality. For example, extracranial bleeding events are usually less serious than Mls or ischemic strokes, particularly in the case of GI bleeding. Although we are not aware of any similar analysis with respect to primary CV prevention, this approach may be considered for studies on contemporary cohorts of subjects, managed with a state-of-the-art control of risk factors and PPI therapy to reduce the risk of $\mathrm{GI}$ bleeding.

\section{Conclusion}

Based on evidence from latest trials, aspirin should no longer be recommended for all patients as part of primary prevention. Following general preventive measures (physical exercise, smoking cessation, treatment of hypertension and hypercholesterolemia, etc.), whose benefits are clear and risks remarkably low, an individualized approach to aspirin prescription is warranted. When patients are less than 70 years of age, clinicians should assess the 10-year CV risk. When that risk is very high and the bleeding risk is low, aspirin treatment should be considered after taking into account the patient's preferences. While the general framework of this approach is quite well defined, the challenges ahead include accurate risk stratification and definition of clear decisional algorithms that could assist clinical cardiologists in delivering the best possible care to their patients.

Conflict of interest: R.D.C. reports personal fees and non-financial support from Bayer, outside the submitted work.
Peer-review: Internally peer-reviewed.

Authorship contributions: Concept - A.A., R.D.C.; Design - R.D.C.; Supervision - None; Funding - None; Materials - None; Data collection and/or processing - None; Analysis and/or interpretation - None; Literature search - A.A., R.D.C.; Writing - A.A.; Critical review - R.D.C.

\section{References}

1. Authors/Task Force Members, Piepoli MF, Hoes AW, Agewall S, Albus C, Brotons C, et al. 2016 European Guidelines on cardiovascular disease prevention in clinical practice: The Sixth Joint Task Force of the European Society of Cardiology and Other Societies on Cardiovascular Disease Prevention in Clinical Practice (constituted by representatives of 10 societies and by invited experts): Developed with the special contribution of the European Association for Cardiovascular Prevention \& Rehabilitation (EACPR). Eur J Prev Cardiol 2016; 23: NP1-96.

2. Gaziano JM, Brotons C, Coppolecchia R, Cricelli C, Darius $\mathrm{H}_{\text {, }}$ Gorelick PB, et al.; ARRIVE Executive Committee. Use of aspirin to reduce risk of initial vascular events in patients at moderate risk of cardiovascular disease (ARRIVE): a randomised, double-blind, placebo-controlled trial. Lancet 2018; 392: 1036-46. [CrossRef]

3. ASCEND Study Collaborative Group, Bowman L, Mafham M, Wallendszus K, Stevens W, Buck G, et al. Effects of Aspirin for Primary Prevention in Persons with Diabetes Mellitus. N Engl J Med 2018; 379: 1529-39. [CrossRef]

4. McNeil JJ, Nelson MR, Woods RL, Lockery JE, Wolfe R, Reid CM, et al.; ASPREE Investigator Group. Effect of Aspirin on All-Cause Mortality in the Healthy Elderly. N Engl J Med 2018; 379: 1519-28.

5. McNeil JJ, Wolfe R, Woods RL, Tonkin AM, Donnan GA, Nelson $M R$, et al.; ASPREE Investigator Group. Effect of Aspirin on Cardiovascular Events and Bleeding in the Healthy Elderly. N Engl J Med 2018; 379: 1509-18. [CrossRef]

6. McNeil JJ, Woods RL, Nelson MR, Reid CM, Kirpach B, Wolfe R, et al.; ASPREE Investigator Group. Effect of Aspirin on Disability-free Survival in the Healthy Elderly. N Engl J Med 2018; 379: 1499-508.

7. Mahmoud AN, Gad MM, Elgendy AY, Elgendy IY, Bavry AA. Efficacy and safety of aspirin for primary prevention of cardiovascular events: a meta-analysis and trial sequential analysis of randomized controlled trials. Eur Heart J 2019; 40: 607-17. [CrossRef]

8. Zheng SL, Roddick AJ. Association of Aspirin Use for Primary Prevention With Cardiovascular Events and Bleeding Events: A Systematic Review and Meta-analysis. JAMA 2019;321: 277-87. [CrossRef]

9. Khan SU, UI Abideen Asad Z, Khan MU, Talluri S, Ali F, Shahzeb Khan $M$, et al. Aspirin for primary prevention of cardiovascular outcomes in diabetes mellitus: An updated systematic review and meta-analysis. Eur J Prev Cardiol 2019: 2047487319825510. [CrossRef]

10. Gaziano JM. Aspirin for Primary Prevention: Clinical Considerations in 2019. JAMA 2019; 321: 253-5. [CrossRef]

11. Marquis-Gravel G, Roe MT, Harrington RA, Munoz D, Hernandez AF Jones WS. Revisiting the Role of Aspirin for the Primary Prevention of Cardiovascular Disease. Circulation 2019; 140: 1115-24. [CrossRef]

12. Chiang KF, Shah SJ, Stafford RS. A Practical Approach to LowDose Aspirin for Primary Prevention. JAMA 2019. [Epub ahead of print] [CrossRef]

13. Capodanno D, Ingala S, Calderone D, Angiolillo DJ. Aspirin for the primary prevention of cardiovascular disease: latest evidence. Expert Rev Cardiovasc Ther 2019; 17: 633-43. [CrossRef] 
14. Ridker PM. Should Aspirin Be Used for Primary Prevention in the Post-Statin Era? N Engl J Med 2018; 379: 1572-4. [CrossRef]

15. Cosentino F, Grant PJ, Aboyans V, Bailey CJ, Ceriello A, Delgado V, et al.; ESC Scientific Document Group. 2019 ESC Guidelines on diabetes, pre-diabetes, and cardiovascular diseases developed in collaboration with the EASD. Eur Heart J 2019; pii: ehz486.

16. Arnett DK, Blumenthal RS, Albert MA, Buroker AB, Goldberger ZD, Hahn EJ, et al. 2019 ACC/AHA Guideline on the Primary Prevention of Cardiovascular Disease: Executive Summary: A Report of the American College of Cardiology/American Heart Association Task Force on Clinical Practice Guidelines. J Am Coll Cardiol 2019; 74: 1376-414. [CrossRef]

17. Valgimigli $M$. The remarkable story of a wonder drug, which now comes to an end in the primary prevention setting: say bye-bye to aspirin! Eur Heart J 2019; 40: 618-20. [CrossRef]

18. De Caterina R, Aimo A, Ridker PM. Aspirin Therapy for Primary Prevention: The Case for Continuing Prescribing to Patients at High Cardiovascular Risk-A Review. Thromb Haemost 2019; doi: 10.1055/ s-0039-3400294. [Epub ahead of print] [CrossRef]

19. Capodanno D, Angiolillo DJ. Aspirin for Primary Cardiovascular Risk Prevention and Beyond in Diabetes Mellitus. Circulation 2016; 134: 1579-94. [CrossRef]

20. Belch J, MacCuish A, Campbell I, Cobbe S, Taylor R, Prescott R, et al.; Prevention of Progression of Arterial Disease and Diabetes Study Group; Diabetes Registry Group; Royal College of Physicians Edinburgh.The prevention of progression of arterial disease and diabetes (POPADAD) trial: factorial randomised placebo controlled trial of aspirin and antioxidants in patients with diabetes and asymptomatic peripheral arterial disease. BMJ 2008; 337: a1840.

21. Fowkes FG, Price JF, Stewart MC, Butcher I, Leng GC, Pell AC, et al.; Aspirin for Asymptomatic Atherosclerosis Trialists. Aspirin for prevention of cardiovascular events in a general population screened for a low ankle brachial index: a randomized controlled trial. JAMA 2010; 303: 841-8. [CrossRef]

22. Aboyans V, Ricco JB, Bartelink MEL, Björck M, Brodmann M, Cohnert T, et al.; ESC Scientific Document Group. 2017 ESC Guidelines on the Diagnosis and Treatment of Peripheral Arterial Diseases, in collaboration with the European Society for Vascular Surgery (ESVS): Document covering atherosclerotic disease of extracranial carotid and vertebral, mesenteric, renal, upper and lower extremity arteriesEndorsed by: the European Stroke Organization (ESO)The Task Force for the Diagnosis and Treatment of Peripheral Arterial Diseases of the European Society of Cardiology (ESC) and of the European Society for Vascular Surgery (ESVS). Eur Heart J 2018; 39: 763-816. [CrossRef]

23. Pickett CA, Jackson JL, Hemann BA, Atwood JE. Carotid bruits as a prognostic indicator of cardiovascular death and myocardial infarction: a meta-analysis. Lancet 2008; 371: 1587-94. [CrossRef]

24. Halvorsen S, Andreotti F, ten Berg JM, Cattaneo M, Coccheri S, Marchioli R, et al. Aspirin therapy in primary cardiovascular disease prevention: a position paper of the European Society of Cardiology working group on thrombosis. J Am Coll Cardiol 2014; 64: 319-27. [CrossRef]

25. Patrignani P, Patrono C. Aspirin and Cancer. J Am Coll Cardiol 2016; 68: 967-76. [CrossRef]

26. [No authors listed]. Aspirin effects on mortality and morbidity in patients with diabetes mellitus. Early Treatment Diabetic Retinopathy Study report 14. ETDRS Investigators. JAMA 1992; 268: 1292-300.

27. Mach F, Baigent C, Catapano AL, Koskinas KC, Casula M, Badimon L, et al.; ESC Scientific Document Group. 2019 ESC/EAS Guidelines for the management of dyslipidaemias: lipid modification to reduce cardiovascular risk. Eur Heart J 2019; pii: ehz455.

28. Williams B, Mancia G, Spiering W, Agabiti Rosei E, Azizi M, Burnier M, et al.; Authors/Task Force Members:. 2018 ESC/ESH Guidelines for the management of arterial hypertension: The Task Force for the management of arterial hypertension of the European Society of Cardiology and the European Society of Hypertension: The Task Force for the management of arterial hypertension of the European Society of Cardiology and the European Society of Hypertension. $J$ Hypertens 2018; 36: 1953-2041. [CrossRef]

29. Expert Panel on Detection, Evaluation, and Treatment of High Blood Cholesterol in Adults. Executive Summary of The Third Report of The National Cholesterol Education Program (NCEP) Expert Panel on Detection, Evaluation, And Treatment of High Blood Cholesterol In Adults (Adult Treatment Panel III). JAMA 2001; 285: 2486-97.

30. D'Agostino RB Sr, Vasan RS, Pencina MJ, Wolf PA, Cobain M, Massaro JM, et al. General cardiovascular risk profile for use in primary care: the Framingham Heart Study. Circulation 2008; 117 : 743-53. [CrossRef]

31. Goff DC Jr, Lloyd-Jones DM, Bennett G, Coady S, D'Agostino RB, Gibbons R, et al.; American College of Cardiology/American Heart Association Task Force on Practice Guidelines. 2013 ACC/AHA guideline on the assessment of cardiovascular risk: a report of the American College of Cardiology/American Heart Association Task Force on Practice Guidelines. Circulation 2014; 129 (25 Suppl 2): S49-73.

32. Knuuti J, Wijns W, Saraste A, Capodanno D, Barbato E, Funck-Brentano C, et al.; ESC Scientific Document Group.2019 ESC Guidelines for the diagnosis and management of chronic coronary syndromes. Eur Heart J 2019; pii: ehz425.

33. Ambale-Venkatesh B, Yang X, Wu CO, Liu K, Hundley WG, McClelland $\mathrm{R}$, et al. Cardiovascular Event Prediction by Machine Learning: The Multi-Ethnic Study of Atherosclerosis. Circ Res 2017; 121: 1092101. [CrossRef]

34. Dimopoulos AC, Nikolaidou M, Caballero FF, Engchuan W, SanchezNiubo $A$, Arndt $H$, et al. Machine learning methodologies versus cardiovascular risk scores, in predicting disease risk. BMC Med Res Methodol 2018; 18: 179. [CrossRef]

35. Tsay D, Patterson C. From Machine Learning to Artificial Intelligence Applications in Cardiac Care. Circulation 2018; 138: 2569-75.

36. Selak V, Jackson R, Poppe K, Wu B, Harwood M, Grey C, et al. Predicting Bleeding Risk to Guide Aspirin Use for the Primary Prevention of Cardiovascular Disease: A Cohort Study. Ann Intern Med 2019; 170: 357-68. [CrossRef]

37. Steering Committee of the Physicians' Health Study Research Group. Final report on the aspirin component of the ongoing Physicians' Health Study. N Engl J Med 1989; 321: 129-35. [CrossRef]

38. Peto R, Gray R, Collins R, Wheatley K, Hennekens C, Jamrozik K, et al. Randomised trial of prophylactic daily aspirin in British male doctors. Br Med J (Clin Res Ed) 1988; 296: 313-6. [CrossRef]

39. Hansson L, Zanchetti A, Carruthers SG, Dahlöf B, Elmfeldt D, Julius $S$, et al. Effects of intensive blood-pressure lowering and low-dose aspirin in patients with hypertension: principal results of the Hypertension Optimal Treatment (HOT) randomised trial. HOT Study Group. Lancet 1998; 351: 1755-62. [CrossRef]

40. Ogawa $H$, Nakayama M, Morimoto $T$, Uemura $S$, Kanauchi M, Doi $\mathrm{N}$, et al.; Japanese Primary Prevention of Atherosclerosis With Aspirin for Diabetes (JPAD) Trial Investigators. Low-dose aspirin for primary prevention of atherosclerotic events in patients with type 2 diabetes: a randomized controlled trial. JAMA 2008; 300: 2134-41. 
41. Ikeda Y, Shimada K, Teramoto T, Uchiyama S, Yamazaki T, Oikawa $S$, et al. Low-dose aspirin for primary prevention of cardiovascular events in Japanese patients 60 years or older with atherosclerotic risk factors: a randomized clinical trial. JAMA 2014; 312: 2510-20.

42. de Gaetano G; Collaborative Group of the Primary Prevention Project. Low-dose aspirin and vitamin $E$ in people at cardiovascular risk: a randomised trial in general practice. Collaborative Group of the Primary Prevention Project. Lancet 2001; 357: 89-95. [CrossRef]
43. [No authors listed]. Thrombosis prevention trial: randomised trial of low-intensity oral anticoagulation with warfarin and low-dose aspirin in the primary prevention of ischaemic heart disease in men at increased risk. The Medical Research Council's General Practice Research Framework. Lancet 1998; 351: 233-41. [CrossRef]

44. Ridker PM, Cook NR, Lee IM, Gordon D, Gaziano JM, Manson JE, et al. A randomized trial of low-dose aspirin in the primary prevention of cardiovascular disease in women. N Engl J Med 2005; 352: 1293-304. 